Comput. Biol. Med. Pergamon Press 1972. Vol. 2, pp. 235-249. Printed in Great Britain.

\title{
Computer Morphometrics in Craniofacial Biology
}

\author{
GEOFFREY F. WALKER \\ and \\ CHARLES J. KOWALSKI \\ Biometrics Laboratory, Dental Research Institute, University of Michigan, \\ Ann Arbor, Michigan, U.S.A.
}

(Received 27 September 1971 and in revised form 13 December 1971)

\begin{abstract}
The quantification, analysis, prediction and simulation of the growth of the craniofacial complex is discussed in the context of a two-dimensional coordinate model of craniofacial morphology. Data accumulated from lateral X-ray films (cephalograms) are used, in conjunction with the model, to describe and analyze craniofacial growth; to predict the future size of craniofacial structures, the future relationships between these structures, the timing of certain structural changes, the velocity and direction of future growth; and to simulate normal, aberrant and constrained growth patterns.
\end{abstract}

\section{INTRODUCTION}

ORGANIZED study of variations in human head form began with the recognition of physical anthropology as a distinct scientific discipline in the early 18 th century. Since that time, anthropologists have employed the methods of classical craniometry (BARNICOT, ${ }^{(1)}$ MONTAGU ${ }^{(2)}$ ) and cephalometry (KROGMAN and SASSOUNI ${ }^{(3)}$ ) to increase their understanding of craniofacial relationships within and between various human populations. In more recent years, workers in dental research have studied normal and abnormal growth and morphology of the dentofacial region with a view towards understanding, and eventual control of, the complex factors associated with malocclusions of the teeth. Since dentitional development is inseparably linked to developmental changes in the craniofacial complex (GRABER ${ }^{(4)}$ ), these parallel lines of inquiry have essentially merged and the resulting field has been labeled craniofacial morphometrics (BLACKITH ${ }^{(5)}$ ). Good summaries of the origins of morphometrics, which extend back to the Pythagorean philosophers of the 5th century B.C., are given by Blackith, ${ }^{(5)}$ RABEY $^{(6)}$ and THOMPSON. ${ }^{(7)}$ More recently, Solow ${ }^{(8)}$ surveyed some of the developments accompanying the marked acceleration in cephalometric research made possible by the advent of the digital computer. In this paper we discuss the approach to cephalometry currently being pursued in the Biometrics Laboratory of the Dental Research Institute at the University of Michigan.

The analysis of craniofacial morphology and growth presents many difficulties. Some of these problems have been discussed by Burstone, ${ }^{(9)}$ ENLOw, ${ }^{(10)}$ FALKNER, ${ }^{(11)}$ HARris, ${ }^{(12)}$ HiRSCHFELD, ${ }^{(13,14)}$ HuXley, ${ }^{(15)}$ JOHNSON, ${ }^{16)}$ KowALSKI, ${ }^{(17)}$ MEDAwar, ${ }^{(18)}$ SCOTT $^{(19)}$ and WALKER et al.,(20,21) to name just a few, but the fundamental difficulty is the inherent complexity of craniofacial morphology and structural change. During growth the face 
undergoes successive changes in which the entire mosaic of its component parts is involved; the enlargement of the craniofacial complex is the cumulative sum of the individual growth increments in all the separate bones of the face as well as those in the cranial base and calvaria. Many studies of this complex process have been made but, with few exceptions, the sample sizes were small with pooled sexes and only a relatively small number of measurements studied. The result has been that the currently used normative patterns of craniofacial morphology and growth are based either on small samples from rather special populations or purely subjective judgements about what constitutes a "good face" and "normal development". Growth increments are all too often inspected by simply superimposing cephalograms (or tracings prepared from these cephalograms) at successive age intervals with little, if any, formal account taken of genetic background, sexual dimorphism, growth potential and other factors known to influence growth dynamics. The entire craniofacial complex is considered as a growing unit because, by visual inspection alone, differentiation of individual components is difficult. This results in an important loss of information since regional growth increments at sutures and on many surfaces may not even be recognized, let alone accurately measured, and because similar end results in total growth can be produced by several fundamentally different combinations of growth activity.

In this paper we describe a two-dimensional coordinate model of craniofacial morphology (WALKER, ${ }^{(22)}$ WALKER and KowALSKI ${ }^{(23)}$ ) and show how it can be used to quantify and study the growth process, to obtain growth projections and to simulate craniofacial growth. Graphically oriented analyses, which clearly depict craniofacial morphology and the ensuing changes during growth, are described and the output from these programs illustrated. The model is bone-surface and suture oriented and consists of the structuring of the cephalogram by a geometrical model which reduces the essential information of the cephalogram into a constellation of coordinate points. This model overcomes many of the difficulties alluded to earlier inasmuch as it introduces a numerical structure so that mathematical manipulations and statistical analyses may be applied, but it retains much of the informational content and descriptive power of the cephalogram itself. In particular, these mathematical manipulations can be used to produce controlled distortions in the model and hence allows the simulation of aberrant growth patterns and of growth changes which would occur under various constraints, e.g. orthodontic appliances, imposed on the model.

\section{THE MODEL}

We describe the model in terms of its implementation in practice, i.e. the steps involved in proceeding from the original cephalogram to the numerical image of this cephalogram as recorded on punched cards or magnetic tape. The first step in the procedure is to trace the original X-ray film on a sheet of transparent matte acetate. The matte acetate is firmly fastened over the surface of the X-ray and the outlines of the skull bones and dentition are traced. The structures actually traced are illustrated in Fig. 1. It is seen that the sagittal projections of the parietal, occipital, frontal, sphenoid and nasal bones, as well as the mandibular and maxillary structures, are included in order to provide a model which allows considerable differentiation of the components of the craniofacial complex.

Tracing is a relatively skilled operation and we have found that it takes several months to train a technician to carry out this procedure with reasonable reliability. We have checked the variation in the tracings from one technician to another and find that there is relative consistency between the tracings of the same technician, but a higher variance between 


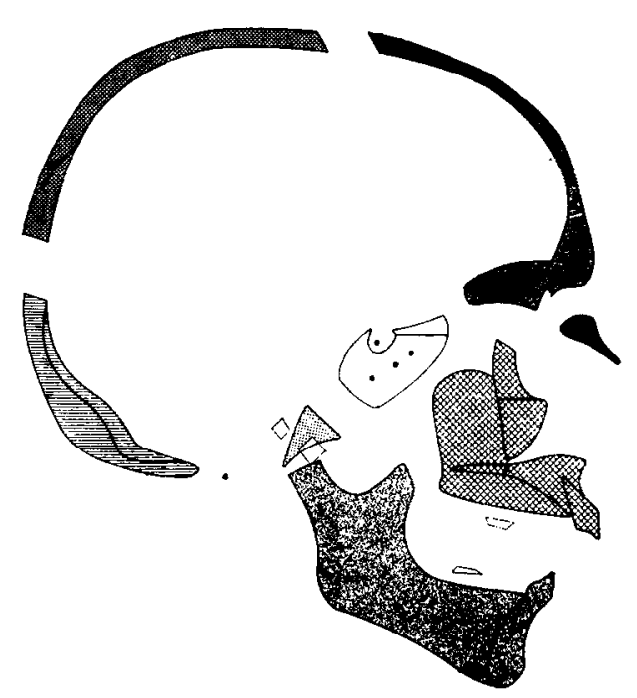

FIG. 1. Components of the dento-facial complex included in our model of craniofacial morphology.

the tracings of two or more technicians. We have introduced quality controls at this stage by carefully checking, and if necessary correcting, the tracings as they are completed. Where possible we try to keep the variation to within $\pm 0.5 \mathrm{~mm}$ for each point comprising the model illustrated in Fig. 2. We have found that many of the points can be seen very clearly on the $\mathrm{X}$-ray and these have relatively low variation; other points are more difficult to locate and these are recorded with a somewhat lower level of confidence. When higher accuracy is required, we repeat the tracings and average the measurements. Of course, it may soon be possible to reduce these recording errors to negligible proportions by the use of more sophisticated scanning equipment: Progress in the area of image processing in the biomedical sciences has been discussed by EAves, ${ }^{(24)}$ LeDley, ${ }^{(25)}$ RAMSEY $^{(26)}$ and Solow ${ }^{(8)}$ and we believe it is now technically possible to automatically scan and extract the relevant information directly from the cephalogram. Indeed, in our opinion, future advances in biometrical processing of cephalometric information are contingent upon the ready availability of this more sophisticated scanning equipment. However, more research and development is necessary before this equipment can be put into routine cephalometric data processing service and thus we are currently using a comparatively slow and cumbersome approach which relies on hand-scanning and semi-automatic digitizing methods as described below.

The next step in the recording procedure is to mark the 177 coordinate points which comprise our mathematical model on the tracing, as illustrated in Fig. 2. The indicated points include all of the traditional anthropometric landmarks (ETTER, ${ }^{(27)}$ KROGMAN and SASSOUNI ${ }^{(3)}$ ) as well as intermediate derived points which are added so that we have a sufficient number of points to reliably describe the shapes of the skull bones. For example, on the sagittal outline of the parietal bones the only well-defined anatomical points are at the extremities, these being Lambda (point \#20) towards the posterior and Bregma (point \#47) at the anterior end. To describe the curve of the skull vault between these two points we divide the parietal curve into eight segments and a point marks the junction of each segment. These derived points are included among those depicted in Fig. 2, which shows the outlines of the skull bones and the 177 points which make up our model. 


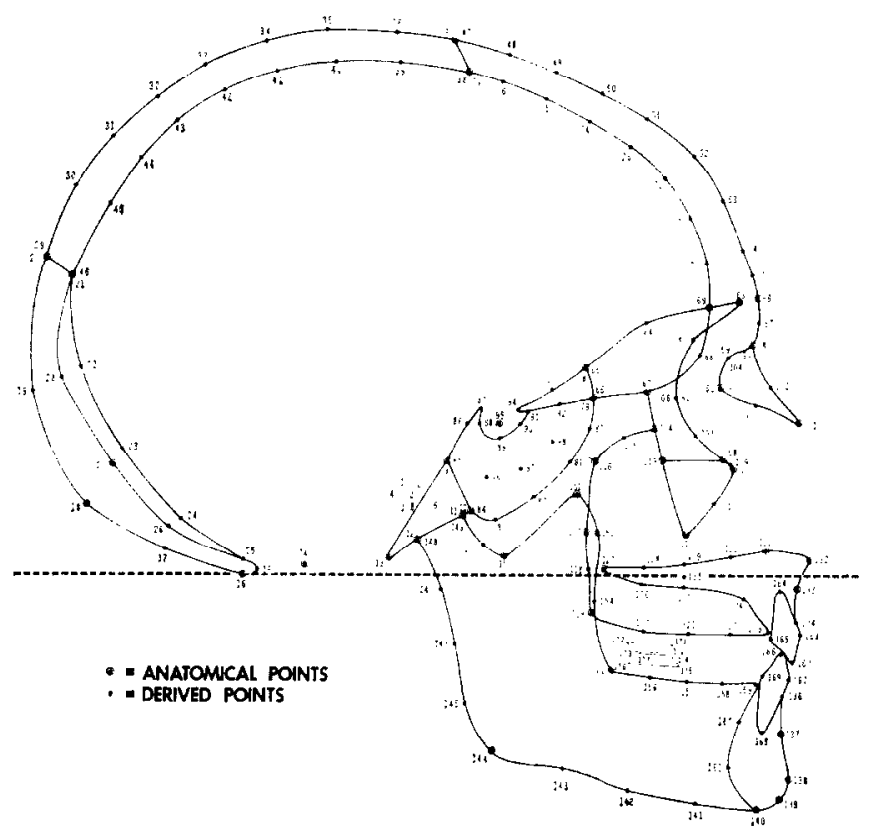

FIG. 2. The 177 coordinate points comprising our model of craniofacial morphology.

The next step is to place the marked tracing on an electronic scanning device and set the machine so that the coordinate axes are centered at a convenient origin and in a convenient orientation. The technician then starts at point $\# 1$ and continues in an unbroken sequence through point $\# 177$, so that the coordinates of all the points are recorded consecutively on punched cards. To operate the machine, two potentiometers are turned so that cross-hairs are brought into position over the marked points and then a recording button is pressed on the digitizing machine. The voltage levels from the two potentiometers (which record the $x$ and $y$ coordinate values as voltage levels) are then transmitted to an analog-to-digital converter and the digital information is fed to a keypunch machine which automatically punches the required coordinate values on cards.

Thus, the measuring and recording procedure consists of three stages: the tracing of the cephalogram, the marking of the 177 points, and the recording of the numerical values of the coordinates of these points on punched cards using an electronic scanning device. The procedure transforms the information contained in the ccphalogram into $x y$-coordinate values which may be stored as cards or converted to magnetic tape and thus be available for processing on a digital computer. An extremely rich data base can now be generated from these coordinate values: Lines, planes, projections, areas, distances and angles are immediately available by the use of coordinate geometry. Statistical analyses can then be carried out on various sets of measurements of interest. Of course, it should be emphasized that the reliability of such analyses is dependent upon the reliability of the underlying measurements (see e.g. Solow ${ }^{(28)}$ and BJÖRK and Solow ${ }^{(29)}$ ). In recognition of this, considerable care is taken with the measuring procedures employed in order to minimize the magnitudes of the measurement errors and, whenever possible, to obtain reliable estimates of these errors. In practice, however, the "acid test" of whether or not the procedure is workable is to check if the recorded coordinates of the model accurately reflect the information contained in the 
tracing and the X-ray film. To check for gross errors we display the recorded coordinates on a cathode ray tube (CRT). More rigorous testing may be accomplished by simply having the computer plot the values for comparison and examples of such plots are given in Figs. 3 and 4.

Here the points are connected by simple straight lines, but other methods of attempting to "reproduce the tracing" from the data banks are certainly possible. These plots are scaled to be comparable to the tracing and X-ray and these may be superimposed to check on the goodness-of-fit. We might remark that by using the 177 points, and straight lines to connect these points, we are essentially able to reproduce the tracing and it is in this sense that we claim that our model retains much of the informational content and descriptive power of the cephalogram. In any event, Fig. 3 illustrates the fact that our plotting programs are not restricted to human skull forms and that we can plot "composite skulls" which are obtained as averages of a number of individual skulls. In Fig. 4 we illustrate the ease with which gross errors are identified and we insist on checking our data bank in this fashion (perhaps on a CRT) before subjecting any portion of it to formal statistical analysis. A more detailed description of our data bank management and manipulation routines is given in the following section.

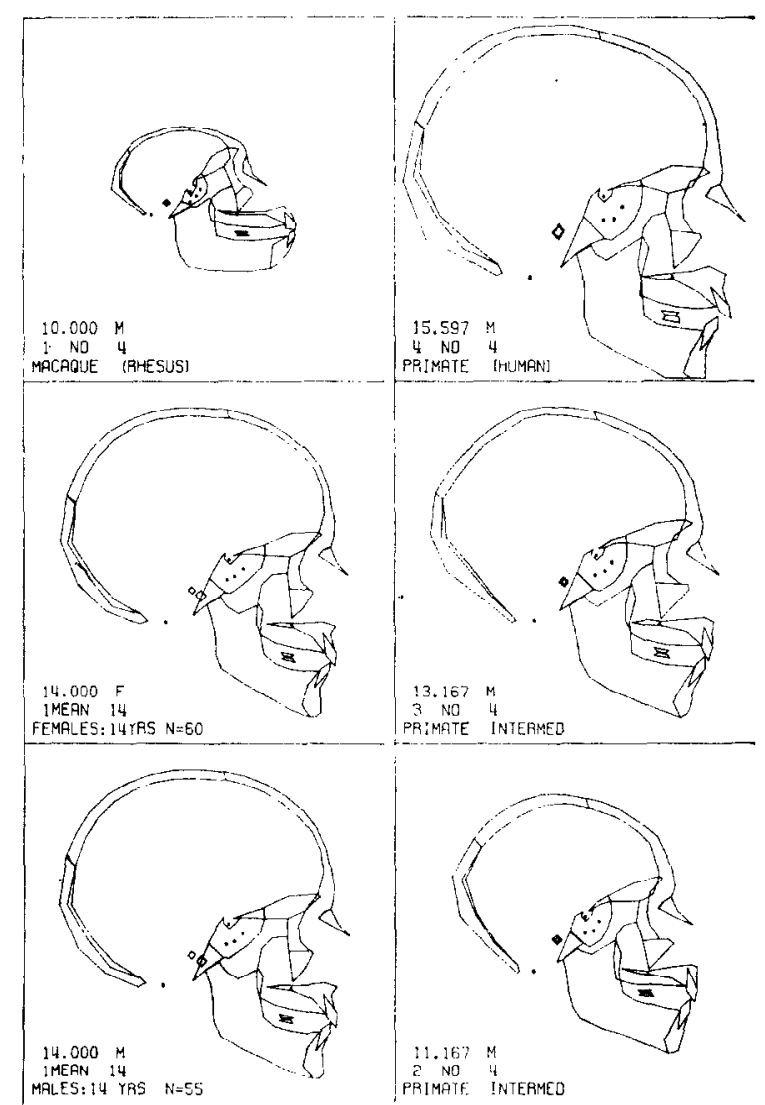

FIr. 3. Computer plots of stored cephalometric coordinate values for lower primate and human subjects. 


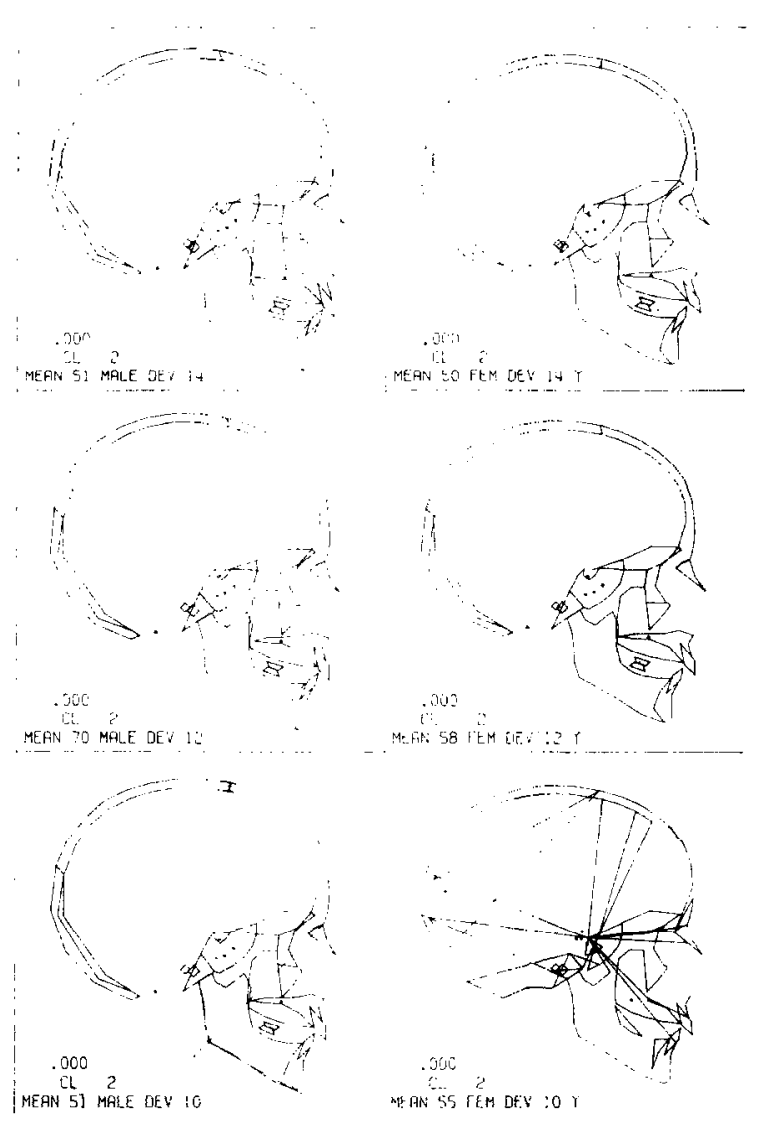

FIG. 4. Computer plots of stored cephalometric mean coordinate values for several groups of individuals.

\section{INFORMATION RETRIEVAL AND VARIABLE EXTRACTION}

In the preceding sections we have described the general problem under consideration, the model to be used in the investigation of this problem, the procedure for transforming the cephalometric information into numerical form and various quality control measures introduced in order to ensure that the data processed in this fashion reliably represents the craniofacial morphology of the subjects included in the study. We turn now to the question of how this data can be accessed by statistical computing programs. This is perhaps not a particularly difficult problem if one has a relatively small amount of data, but we currently have over 30,000 records, each record containing the coordinates of the 177 points along with appropriate demographic information and identification codes, in our data banks. These include individuals of various races, sexes, ages, genetic backgrounds, etc. as well as a number of orthodontic case histories, with cephalograms taken before, during and after treatment. The data for certain groups is cross-sectional, for others its longitudinal and for certain groups we have mixed cross-sectional and longitudinal data. It is not our purpose here to describe in detail our data management and manipulation routines, but rather to indicate what sorts of manipulations are possible and to point out that in a system of this magnitude a considerablc amount of pre-processing is necessary before one can analyze 
selected portions of the data. In particular, one must be able to form subgroups of the data bank and to extract the variables he wishes to study since most standard analysis programs require the delineation of the groups of subjects to be analyzed as well as a variable list. We have developed console-oriented, user-prompting programs which can form groups on the basis of a number of criteria (e.g. we can access all the white American boys between 12 and 14 years of age having "normal" dental occlusions) and which can extract any variable definable in the context of our model of craniofacial morphology (Fig. 2). These data are then copied into files which can be accessed by a variety of statistical computing programs. Thus Fig. 5 shows the histogram of the ANB angle (the angle defined by the points \#133, \#58 and \#137) for $N=113$ white American males between 12 and 14 years of age having normal dental occlusions.

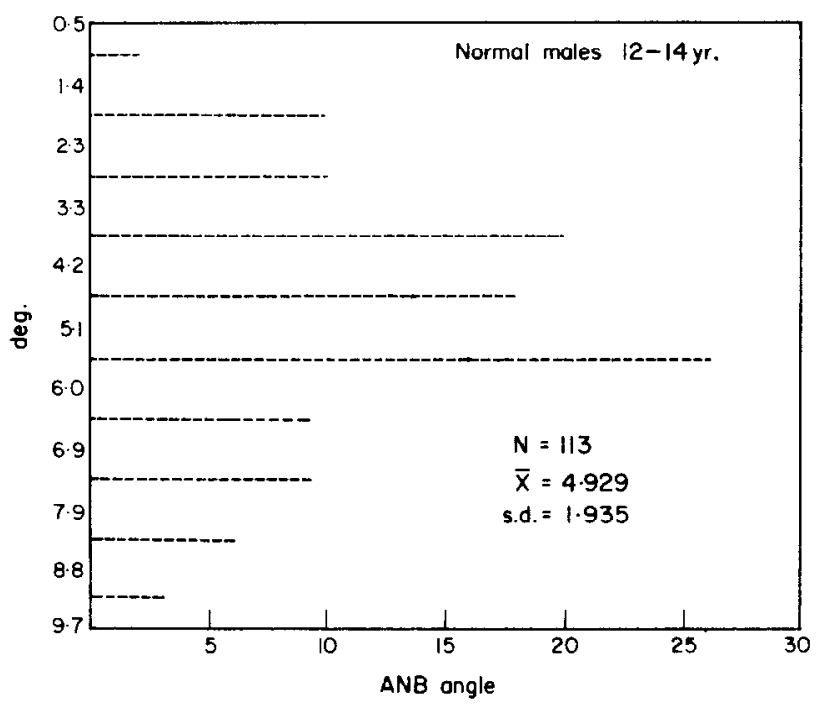

FIG. 5. Histogram of the ANB angle for 113 normal males from 12-14 years of age.

Cross-sectional growth curves for the ANB angle for "normal" males and females from 6 to 24 years of age, with approximate 95 per cent confidence intervals for the mean ANB angle in each age bracket, are shown in Fig. 6. A more detailed description, analysis and interpretation of these data is given by WALKER and KowALSKI ${ }^{(30)}$ and other variables were considered in other investigations. ${ }^{(31-33)}$ An analysis of a subset of thirteen variables in several groups was presented by HARRIS, KowALSKI and WALKER. ${ }^{(34)}$ The important point to be made here is that the model illustrated in Fig. 2, given the appropriate software, allows the investigator to extract and examine individual variables, or sets of variables, yet retains much additional information which may be accessed for other purposes. An important benefit is the ability of the investigator to view the results and interpret the variations as a unified whole (all the structures depicted in Fig. 1 are available) in a medium which is readily understood by all.

In the next section we describe our approach to the study of the growth pattern of the entire collection of coordinate points. We feel that such a study is more valuable than a study concentrating on only a selected number of linear and/or angular variables inasmuch as the growth of each of the components of the craniofacial complex can be followed, allowing 


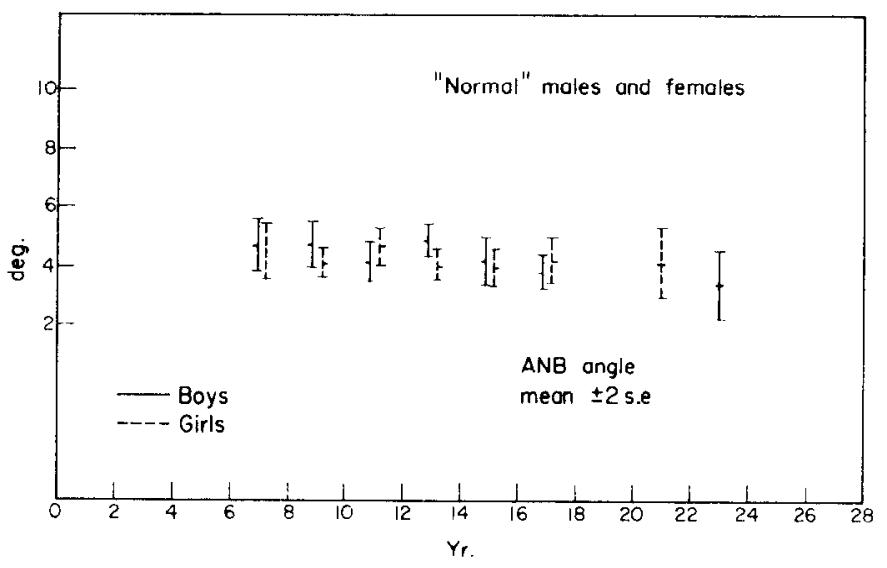

FIG. 6. Sex-specific growth curves for the ANB angle.

a more detailed consideration of changes in shape than is possible using traditional methods. However, should one wish to follow the growth of any derived measurement, e.g. mandibular length, this variable is easily extracted from the model and its growth may be studied by conventional procedures.

\section{VECTORGRAMS OF GROWTH}

A vectorgram is, simply, the observed growth trajectory associated with any one of the 177 points comprising the model. Let the position of point $j(j=1, \ldots, 177)$ for individual $i(i=1, \ldots, N)$ at time $t_{k}\left(k=1, \ldots, K_{i}: t_{1}<\ldots<t_{K_{i}}\right)$ be denoted by

$$
P_{i j}\left(t_{k}\right)=\left[X_{i j}\left(t_{k}\right), y_{i j}\left(t_{k}\right)\right]
$$

Then the vectorgram of the $i^{\text {th }}$ individual for point $j$ is denoted by

$$
V_{i j}=\left[P_{i j}\left(t_{1}\right), \ldots, P_{i j}\left(t_{K_{i}}\right)\right]
$$

and the vectorgram set for individual $i$ is defined as

$$
V_{i}=\left[\begin{array}{l}
V_{i j} \\
\cdot \\
\cdot \\
\cdot \\
V_{i, 177}
\end{array}\right]
$$

The vectorgram set for a given individual completely describes the observed growth trajectories for each of the 177 points, but this model is a relatively complicated one for evaluating change and predicting growth. In HoRsT's ${ }^{(35)}$ terminology, we have sets of data matrices on each of a number of attributes (the $x y$-coordinate values), for each of a number of entities (individuals), for each of a number of conditions (genetic and/or environmental groupings), taken on a number of different occasions. Horst surveyed the problems associated with the analysis of models of this general structure and concluded that, "The general problem is extremely complex and no adequate solutions are currently available". Even if we concentrate on a single individual and a particular one of the 177 points we have what amounts to a bivariate time series where, e.g. the true growth trajectory may be assumed to be a polynomial or some other function of time. But the analysis of the data 
by ordinary time series methods ${ }^{(13,14)}$ is complicated by the fact that the time points will not in general be equally spaced and, when more than one individual is considered, we may have very different observation time sequences $t_{1}, \ldots, t_{K_{i}}$ to contend with and, often, individuals will have different numbers of total observations. Nor is it clear in the two-dimensional case how one can mirror the pubertal spurt and other characteristic features of growth curves (TANNER ${ }^{(36)}$ ) in terms of a tractable bivariate function of time. In an attempt to circumvent these difficulties we have, as a first approximation at least, considered titting the observed $x$-coordinate increments to time, say $x=g(t)$, and then assuming that the corresponding $y$-values are given by a polynomial in $x=g(t)$. Fitting the polynomial by least squares we have found that a quadratic function generally describes the data quite well, the coefficients in the regression equation being estimated by combining vectorgrams for similar individuals. The resultant regression equations

$$
x=g(t), y=a x^{2}+b x+c
$$

constitute what we call the composite vectorgram when the vectorgrams of several individuals are used to estimate the quadratic regression of $y$ on $x$; when only a single individual is involved we refer to the regression equations as the smoothed vectorgram. This formulation of the problem is tractable inasmuch as growth curves $g(t)$, as well as their derivatives $g^{\prime}(t)$, for marginal growth and rate of change of growth in the $x$-direction are readily available and can be used to interpolate within (and extrapolate beyond) various observation time sequences $t_{1}, \ldots, t_{K_{i}}$ and to reflect the pubertal spurt as well as other features of the typical (marginal) growth curve. The fitting of $y$ as a quadratic in $x$ can then be thought of as simply a device for smoothing vectorgrams or for producing smoothed composite vectorgrams. These composite vectorgrams can then be assembled for each of the 177 points for some group of individuals and growth projections for similar individuals obtained. It might be noted at this point that the use of vectorgrams in practice is complicated by the fundamental problem of determining which structures in the skull are to be used for orientation of the successive records of the same subject. Indeed, the observed growth trajectories depend on the choice of the origin. In recognition of this problcm, wc have written our programs so that any point may be designated as fixed and the observed growth increments are, then, relative to the orientation chosen. The solution of the general problem requires further investigation but, in the meantime, the investigator is free to choose the orientation on grounds of convenience or "in accordance with the purposes of the investigation". In what follows we assume that this choice has been made and the discussion is confined to the fixed coordinate space selected.

\section{GROWTH PROJECTIONS; MEAN VALUE SIMULATION}

Suppose we are given a group of $N$ homogeneous individuals and serial cephalograms for each. We can then average the vectorgrams of these individuals for each of the 177 points (or for some suitable subset of these points) to obtain a set of smoothed composite vectorgrams and the associated confidence bands about these composite vectorgrams. Then given a new individual of the same type we could, in the absence of any additional a priori information, obtain a growth projection by simply assuming that this new individual will grow according to the mcan growth trajectory of his peer group. As growth proceeds, the actual and predicted values may be compared and updated projections obtained. Thus it is possible to simulate growth activity using the averages of a number of like individuals (this is called 
an "expected value simulation model" by MARTIN(37)), giving an indication of what the child could be expected to look like if he were to follow the average growth pattern of his peers. A simplistic illustration is given in Fig. 7. Growth trajectories associated with point \#138 (Pogonion) are plotted for seven different individuals from 8 to 12 years of age.

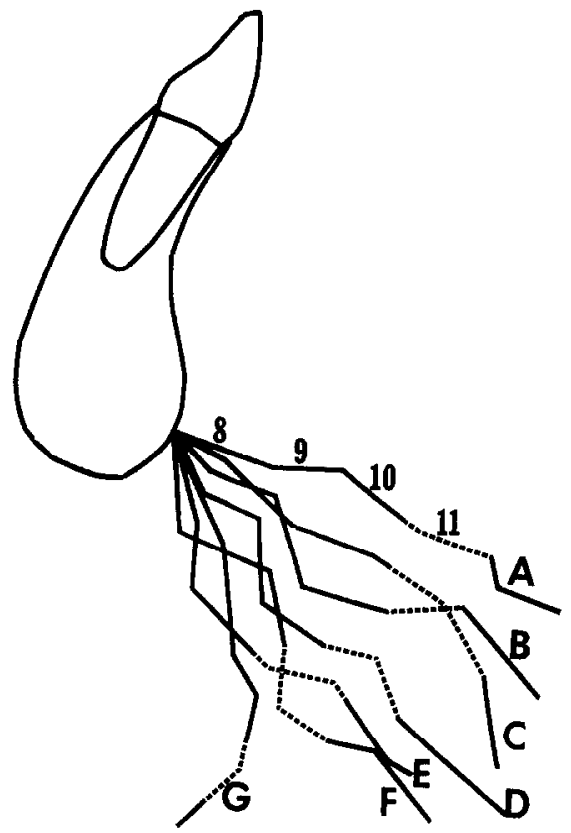

FIG. 7. Growth trajectories for pogonion for several individuals.

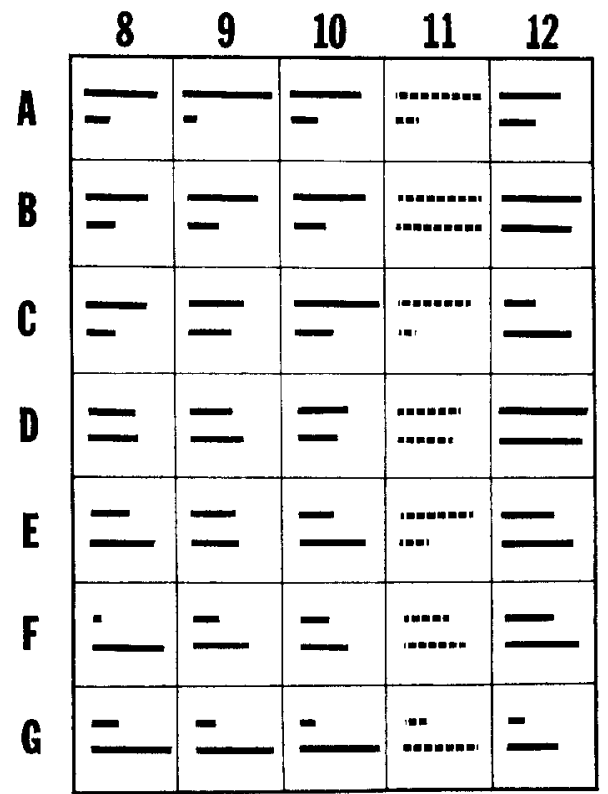

FIG. 8. Marginal growth increments for pogonion for several individuals. 
Figure 8 shows the corresponding marginal growth increments for each of the seven subjects during each of the five years of observation; the $x$-increments are shown above the $y$-increments in each cell of the table, and a glance at this table gives a useful indication of both the direction and velocity of the growth of the point Pogonion. For example, subject $A$ has a strong horizontal component of growth; subject $D$ is growing in the more typical south-east direction; subject $\mathrm{G}$ is exhibiting a strong vertical growth tendency. These data may then be averaged and the corresponding annual growth increments plotted to give the investigator some insight into the structural changes that accompany "average growth". In Fig. 9, for example, a smoothed vectorgram subset (not all of the 177 average growth trajectories are shown) which may be used to predict the average growth of 14-year-old females during the next year is given. The "average skull" allows the investigator to assess where any particular subject stands relative to the norm at any given point in time and the average growth trajectories allow some degree of growth prediction to be made. If several $\mathrm{X}$-rays of the individual for which the projection is desired are available, so that one can assess the relative magnitudes of the horizontal and vertical components of the resultant vector of growth, more precise predictions are of course possible. The key to the success of a prediction system of this kind is that it be dynamic: If a child appears to be an early maturer, the timing of the pubertal spurt should be adjusted in the regression cquation $x=g(t)$; if more than one X-ray is available, this information should be incorporated into the prediction equations. Very few individuals can be expected to follow the average growth trajectory for the group and provision should be made in the prediction system to incorporate additional information as it becomes available. Given a single X-ray, one can do no better than to base his prediction on the mean growth trajectories of a peer group, and such estimates are typically unreliable, but as serial information is accumulated the use of conditional mean values becomes possible and may be expected to lead to more reliable estimates.

Figure 9 can, of course, be extended to show more than a single growth increment. Figure 10 shows the average growth pattern for the entire constellation of points (again, these are

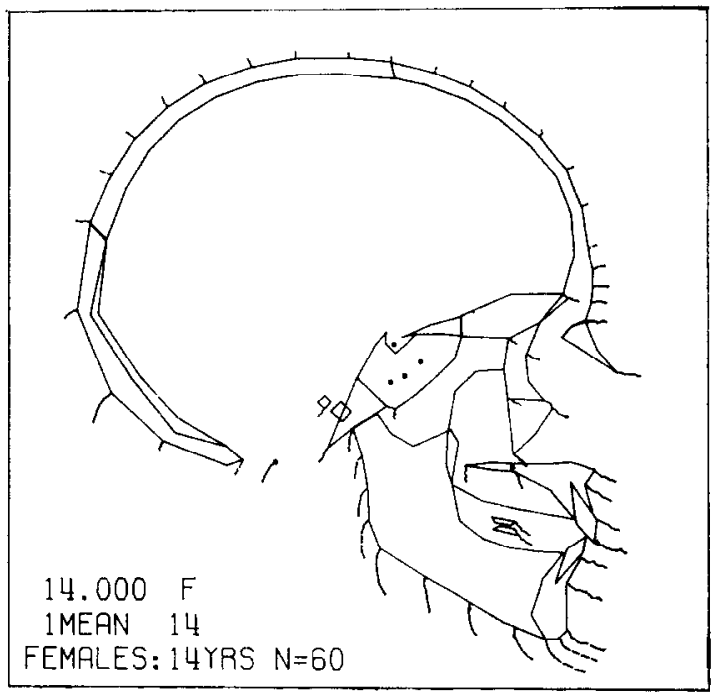

FIG, 9. Smoothed vectorgram subset for a group of 14-year-old females. 
connected by simple straight lines) for several years. Plots of this kind are of special interest to orthodontists inasmuch as they clearly reveal the predicted future size of the craniofacial structures, the future relationships between these structures, the timing of certain structural changes, and the velocity and direction of future growth.

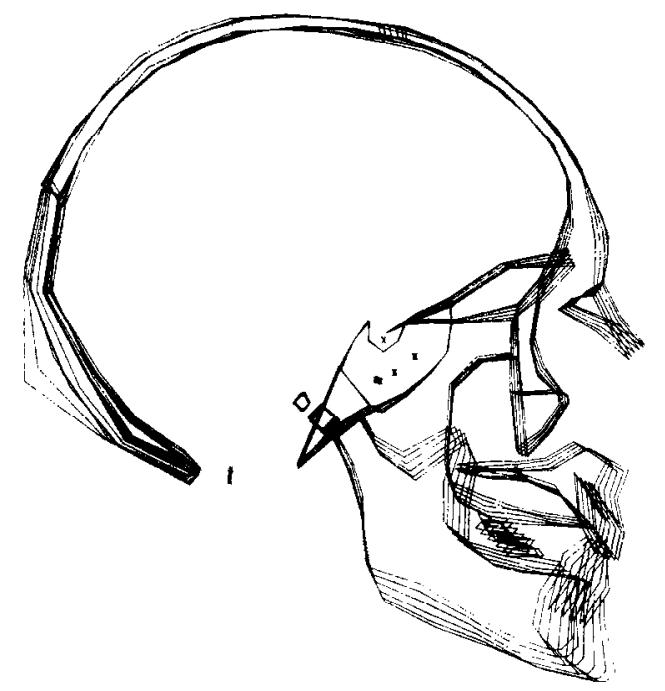

Fig. 10. Projected growth pattern for several years.

\section{SIMULATION OF ORTHODONTIC TREATMENT}

If the expected growth pattern of a given individual promises to produce or maintain an existing malocclusion and/or facial imbalance, orthodontic treatment may be initiated in an attempt to channel the growth activity into a more favorable pattern. For example, to treat a class II malocclusion (a condition in which the upper teeth bite anterior to the lower teeth), the strategy calls for restricting the horizontal growth of the maxilla and/or developing the mandible forward. However, there are a number of ways to accomplish this goal: There has always been a marked difference of opinion as to what constitutes correct and effective therapy for the mandible (STEINER $\left.{ }^{(38)}\right)$. These opinions vary from simply "jumping the bite" to more elaborate appliance procedures aimed at altering mandibular size, shape and location. One way of deciding between the available alternatives, if one has enough data on the oulcomes of such treatment regimens, is to use our model to estimate the effects of these different treatments on the given individual. If such data are not available, one might attempt to adjust the parameters in the regression system $x=g(t)$ to mirror the constrained horizontal growth of the maxillary structures and the enhanced horizontal growth of the mandible appropriate for the various available treatments. And horizontal growth control is crucial: As stated by GRABER, ${ }^{(4)}$ "To the orthodontist treating a class II malocclusion characterized by a mandibular retrusion, the pubertal growth spurt, with its emphasis on the horizontal vector of growth, may mean the difference between restoration of a normal jaw relationship and a residual class II mandibular retrusion". Once the usual rates and locations of growth become known, and their dependence on skeletal age and maturation is 
evaluated, the realistic simulation of craniofacial growth becomes possible and such studies can be expected to lead to accurate prediction of growth and to provide a sound basis for indicating the best timing for orthodontic treatment, hence potentially increasing the efficiency and decreasing the duration of treatment.

The social impact of such a system is potentially great. In the United States, approximately one-third of the school age population suffer from malocclusions severe enough to require orthodontic treatment. Thus, in 1968, some 14 million children could have benefitted from such care. However, the 4000 orthodontists in practice that year could treat less than 10 per cent of this group. It is clear that we need to develop tools to improve and extend treatment, as well as more well-trained orthodontists. Since surveys show (GRABER ${ }^{(4)}$ ) that two-thirds of the patients seen for orthodontic therapy have types of malocclusion in which growth and development play a significant role in the success or failure of treatment, one such tool might be the model discussed in this paper. Simulation may represent another such tool. The ability to simulate the growth patterns associated with the development of various types of malocclusion and the effects of orthodontic treatment enables the clinician to synthetically treat a given case in a number of ways and hence to discover the possibilities and limitations of the available treatments. Simulation may also be useful as a teaching aid, enabling the student to use a simulation program to "treat" many more cases in a shorter period of time than is otherwise possible.

While much work remains to be done if we are to realize the potentialities of this system, our early results have convinced us that our approach is feasible. Consider, for example, the situation depicted in Fig. 11. Here the projected growth pattern indicates the maintenance of a class III malocclusion (a condition in which there is a marked projection of the mandible, resulting in the lower teeth biting anterior to the maxillary teeth) and the treatment strategy revolves around restricting the horizontal growth of the mandibular structures. The aim is to have future growth result in a favorable occlusion, say as shown in Fig. 10. The problem is to match the growth functions $x=g(t)$ necessary to produce the desired effect with one of the

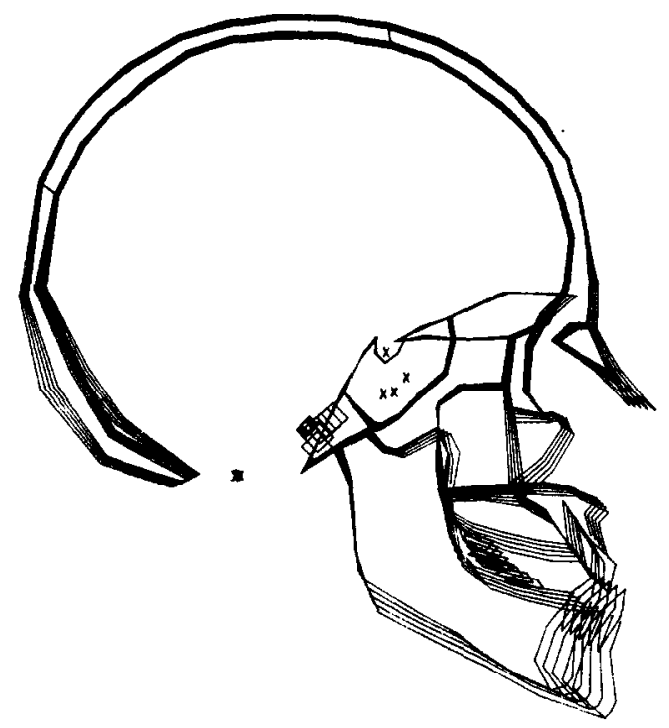

FIG. 11. The growth pattern associated with a class III malocclusion. 
available treatments or to choose that treatment which, on the basis of experience with similar cases, can be expected to channel the growth activity into the most favorable pattern.

We are currently in the process of preparing a large volume of data on orthodontic case histories. We have already prepared a large volume of normative data collected over a period of some twenty years by Dr. W. M. Krogman at the Philadelphia Center for Research in Child Growth, and some of these data were used in the examples considered in this paper. Our aim is to amalgamate this information to form a dynamic system of diagnosis which considers the patient's progressive growth changes, evaluates his facial growth potential against norms for his age and maturation, and plots and tabulates the expected growth increments for the ensuing years under various treatment regimens and, in effect, provides the framework of evidence within which an effective treatment plan may be designed. Our progress in this direction has been discussed and, while more sophisticated scanning equipment would serve to facilitate our collection of data, the ultimate tool-the computer-has served us well.

\section{SUMMARY}

The quantification, description, analysis, prediction and simulation of the growth of the craniofacial complex is discussed in the context of a two-dimensional coordinate model of craniofacial morphology. Data accumulated from lateral X-ray films (cephalograms) are used, in conjunction with the model, to derive measurements of the growth of the face with respect to rate, direction, location and timing, producing normative patterns of growth for various population groups. The method requires the reduction of a lateral head X-ray film to a line drawing (tracing) of the bone contours. The tracing is then reduced to a constellation of 177 points which describe the shape, size and position of the craniofacial bones. These points are recorded as $x y$-coordinates using an electronic scanning device which converts the coordinates to numbers on punched cards and hence into form directly amenable to the storage, retrieval and manipulation of craniofacial growth information.

Graphically oriented analyses, which clearly depict craniofacial morphology and the ensuing changes during growth, are described and the output from these programs illustrated. We show how to measure growth changes and how to quantify and record individual growth patterns so that they may be displayed and compared with normative patterns of growth. It is possible to derive equations to fit this growth data and to compute the position of any point (or derived measurement) in the model at any selected age. It is also possible to extrapolate the growth curves (using regression or time-series methods) and to provide some degree of growth prediction. Finally, it is possible to estimate orthodontic forces and the resultant dental and facial changes in a series of patients and to develop programs to recreate the corresponding changes in the model by means of the computer. This has obvious clinical importance, but may also be recognized as a useful teaching device allowing the student to see the (estimated) long-term effects of a particular treatment in a matter of seconds.

\section{REFERENCES}

1. N. A. BARnicot, Biological variation in modern populations, Human Biology, G. A. HARRISON, J. S. Weiner, J. M. Tanner and N. A. Barnicot. Oxford Univ. Press, Oxford (1964).

2. M. F. A. Montagu, A Handbook of Anthropometry. Thomas, Springfield, Ill. (1960).

3. W. M. Krogman and V. SAssouni, A Syllabus in Roentgenographic Cephalometry. Philadelphia Center for Research in Child Growth, Philadelphia (1957).

4. T. M. Graber, Craniofacial and dentitional development, Human Development, F. FALKNER, ed. W. B. Saunders, Philadelphia (1966). 
5. R. E. BlackIth, Morphometrics, Theoretical and Mathematical Biology, T. H. WATERman and H. J. Morowitz, eds. Blaisdell, New York (1965).

6. G. RABEY, Morphanalysis. H. K. Lewis, London (1968).

7. D'A. W. Thompson, On Growth and Form, 2nd rev. ed. Cambridge Univ. Press, London (1942).

8. B. Solow, Computers in cephalometric research, Comput. Biol. Med. 1, 49 (1970).

9. C. J. Burstone, Process of maturation and growth prediction, Am. J. Orthodont. 49, 907 (1963).

10. D. H. Enlow, The Human Face. Harper and Row, New York (1968).

11. F. FALKNER, General considerations in human development, Human Development, F. FALKNer, ed. W. B. Saunders, Philadelphia (1966).

12. J. E. HARris, Problems in the statistical inspection of craniofacial variables during growth and development, Cranio-Facial Growth in Man, R. E. Moyers and W. M. Krogman, eds. Pergamon Press, Oxford (1971).

13. W. J. HIRSCHFELD, Time series and exponential smoothing methods applied to the analysis and prediction of growth, Growth 34, 129 (1970).

14. W. J. HrrschFeld, A comparison of regression with time series exponential smoothing predictions of craniofacial growth, Growth 34, 431 (1970).

15. J. HuXLey, Problems of Relative Growth. Methuen, London (1932).

16. L. Johnson, A statistical evaluation of cephalometric prediction, Angle Orthodont. 38, 284 (1968).

17. C. J. KowALSKI, A commentary on the use of multivariate statistical methods in anthropometric research, Am. J. Phys. Anthrop. 36, 119-131 (1972).

18. P. B. Medawar, Size, shape and age, Essays on Growth and Form, W. E. Le Gros Clark and P. B. Medawar, eds. Oxford Univ. Press, Oxford (1945).

19. J. H. ScotT, Dento-facial Development and Growth. Pergamon Press, Oxford (1967).

20. G. F. WALKER and C. J. KowALSKI, Simulation of craniofacial growth, Proceedings of the 1971 Summer Computer Simulation Conference, Vol. II, p. 1014. Board of Simulation Conferences, Denver (1971).

21. G. F. Walker, R. Grainger, W. S. Hunter, R. Ledley and F. Westervelt, New techniques in handling growth data, Cranio-facial Growth in Man, R. E. Moyers and W. M. Krogman, eds. Pergamon Press, Oxford (1971).

22. G. F. WALKER, Summary of a research report on the analysis of cranio-facial growth, New Zealand Dent. Jl 63, 31 (1967).

23. G. F. WALkER and C. J. KowALSKI, A two-dimensional coordinate model for the quantification, description, analysis, prediction and simulation of craniofacial growth, Growth 35, 191 (1971).

24. G. N. EAves, Image processing in the biomedical sciences, Comput. Biomed. Res. 1, 112 (1967).

25. R. S. LEDLEY, High-speed automatic analysis of biomedical pictures, Science 146, 216 (1964).

26. D. M. Ramsey, ed., Image Processing in Biological Science. Univ. California Press, Berkeley (1968).

27. L. E. ETter, Atlas of Roentgen Anatomy of the Skull. Thomas, Springfield, Ill. (1955).

28. B. Solow, The pattern of craniofacial associations, Acta. Odont. Scand. 24, Suppl. 46 (1966).

29. A. BJork and B. Solow, Measurement on radiographs, J. Dent. Res. 41, 672 (1962).

30. G. F. WALKER and C. J. KowALSKI, A cephalometric investigation of the distribution of the ANB angle in a large sample of normal individuals, Angle Orthodont. 41, 332 (1971).

31. C. J. KowalSki and G. F. WALKer, Distribution of the mandibular incisor-mandibular plane angle in "normal" individuals, J. Dent. Res. 50, 984 (1971).

32. C. J. Kowalski and G. F. WALker, The Tweed triangle in a large sample of normal individuals, J. Dent. Res. 50, 1690 (1971).

33. G. F. WALKER and C. J. Kowalski, On the growth of the mandible. Am. J. Phy's. Anthrop. 36, 111-117(1972).

34. J. E. HARRIS, C. J. KowAlski and G. F. WALKER, Discrimination between normal and class II individuals using Steiner's cephalometric analysis, Angle Orthodont. In press. (To appear in July, 1972).

35. P. Horst, Multivariate models for evaluating change, Problems in Measuring Change, C. W. Harris, ed. Univ. Wisconsin Press. Madison (1963).

36. J. M. Tanner, Human growth and constitution, Human Biology, G. A. Harrison, J. S. Weiner, J. M. TANNER and N. A. Barnicot. Oxford Univ. Press, Oxford (1964).

37. F. F. MARTIN, Computer Modeling and Simulation, p. 5. Wiley, New York (1968).

38. C. C. SteIner, Cephalometrics in clinical practice, Angle Orthodont. 29, 8 (1959). 